\title{
Influence of Reference Bed Level on Computation of Bed Shear Stress in Open Channel Flow
}

\author{
K.P.P. Pathirana, P.C. Ranasinghe and U.R. Ratnayake
}

\begin{abstract}
An accurate estimation of bed shear stress is essential for estimating sediment transport rates in streams, rivers and open channels. Precise velocity measurements were carried out in steady, uniform open channel flows over a rough bed with large scale roughness to compare different methods of estimating bed shear stress. Effect of reference bed level for measurement of water depth which is an important parameter for computing bed shear stress, was investigated.
\end{abstract}

Keywords: Bed shear stress, Shear velocity, Roughness height, Reference bed level

\section{Introduction}

The sediment transport in alluvial channels and rivers has attracted civil engineers for a long time. The knowledge of sediment transport rate is important as it directly or indirectly influences many problems such as the prediction of natural changes of river morphology, dredging of sediment deposits, the estimation of the lifetime of reservoirs and the prediction of pollutant concentrations at combined sewer outfalls etc. Bed shear stress is one of the key parameters required in estimating the sediment transport rates in streams, rivers and open channels. Hence, an accurate estimation of bed shear stress is essential. The determination of actual bed level is reported to be one of the difficulties when computing bed shear stress in uniform flows over a rough bed with large-scale roughness. This paper investigates the effect of bed level on computation of bed shear stress in steady, uniform open channel flows using laboratory experiments conducted in an open channel with a fixed rough bed.

\section{Computation of Bed Shear Stress}

The computation of bed shear stress in steady, uniform open channel flow is studied through shear velocity of the flow as it is directly related to bed shear stress.

The shear velocity can be calculated using four different methods described below. The effect of reference bed level on shear velocity is much more pronounced in the first two methods, as water depth is necessary to compute shear velocity in these two methods. In methods $3 \&$ 4 , the computation of shear velocity is based on hydraulic radius and therefore, direct influence of reference bed level on shear velocity computation is comparatively low.

\section{METHOD 1:}

For a hydraulically rough bed (Figure 1), velocity distribution over the water depth in terms of shear velocity is given by the following logarithmic equation (Chien et al., [2]),

$$
\frac{u}{u_{.}}=\frac{1}{\chi} \ln \left(\frac{y+\Delta y}{k_{s}}\right)+B_{s}
$$

where, ks = equivalent roughness height, $\|=$ velocity, $y=$ water depth, $u^{*}=$ shear velocity, $x=$ Von Karman coefficient, $\Delta y=$ distance between the bed and the reference bed level where the velocity is zero (hypothetical bed) and Bs = a coefficient. For large scale roughness, the hypothetical bed level is located in between the bottom and the maximum height of the non-uniform roughness elements as shown in Figure 1. $u^{*}$ is obtained using Eq.(1) from the gradient of the plot of $u$ vs. $\ln (y)$ for the near-bed velocity measurements. The equivalent roughness height, $\mathrm{k}_{\mathrm{s}}$ is computed using the intercept of the same plot.

\section{METHOD 2:}

In this method, the same logarithmic velocity distribution (Eq.1) is used taking the reference

Eng.(Dr.) K.P.P. Pathirana. B.Sr. Eng. (Hons.KPeradeniya). M.Eng.. Ph.D., C.Eng.. FIE(SL). MICE. Senior Lecturer. Deparment of Civil Engineering. University of Peradeniya.

Eng. P.C. Ranasinghe. B.Sr. Eng. (Hons.), (Perndeniyn), AMIE(SL), Civil Engineer, Rand Development Authority:

Eng.(Dr.) U.R. Ratmayake. . B.Sr. Eng. (Hons). (Peradeniyn), M.Eng., D.Eng. (AIT. Bangkok), C.Eng.. MIE(SL).

Senior Lecturer. Department of Civil Engineering. University of Peradeniya. 
bed level as the datum, which passes through sand grains at a height of the average size of particles $\left(d_{50}\right)$. The shear velocity is computed using the gradient of the plot of $u$ vs. $\ln (y)$ for near bed velocity profile and roughness height using the intercept.

\section{METHOD 3:}

The relationship between mean velocity and $\mathrm{u}^{*}$ is given by (Chang, [1]);

$$
\frac{U}{u_{v}}=6.25+5.75 \log \left(\frac{R_{h}}{k_{s}}\right)
$$

where $U=$ mean velocity and $R_{h}=$ hydraulic radius. The shear velocity is calculated using the mean velocity of the flow at a particular section using equation (2) with the average value of $k_{s}$ obtained from methods 1 and 2 .

\section{METHOD 4:}

The shear velocity for steady uniform flow is given by;

$$
u_{*}=\sqrt{g R_{h} S}
$$

where, $R_{h}=$ hydraulic radius, corrected for sidewall effects and $S=$ channel bed slope.

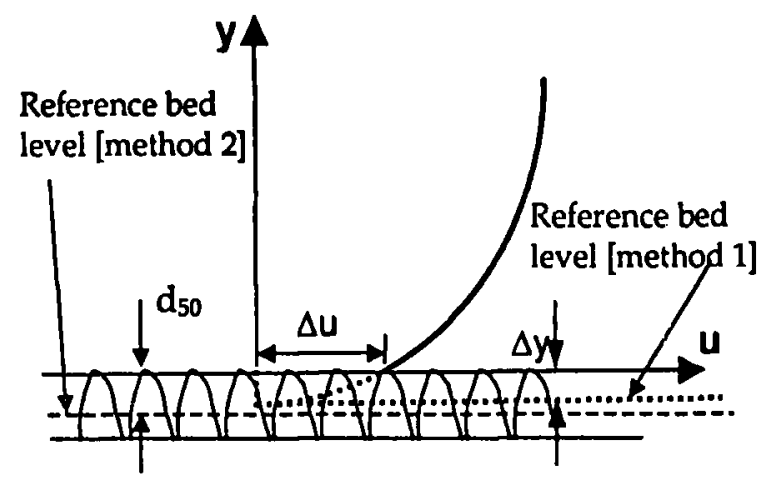

Figure 1 - Definition of reference bed level

\section{Experimental Set-up}

The experiments were carried out in a $10 \mathrm{~m}$ long, $0.4 \mathrm{~m}$ wide, $0.5 \mathrm{~m}$ deep rectangular, recirculating, tilting flume in the Hydraulics Laboratory of the Faculty of Engineering, University of Peradeniya. A schematic diagram of the experimental set-up is shown in Figure 2. The upstream tank is $1.5 \mathrm{~m}$ long, $1 \mathrm{~m}$ deep and 1 $\mathrm{m}$ wide and it is shaped to gradually narrow in width at the end to fix with the channel entrance, in order to avoid any undesired sudden undulations of the flow. A sharp crested weir is fixed at the end of the channel with a depth gauge to facilitate the estimation of flow discharges. The special features of the set-up such as, sediment trap and load cell were not used in the present study as all the experimental

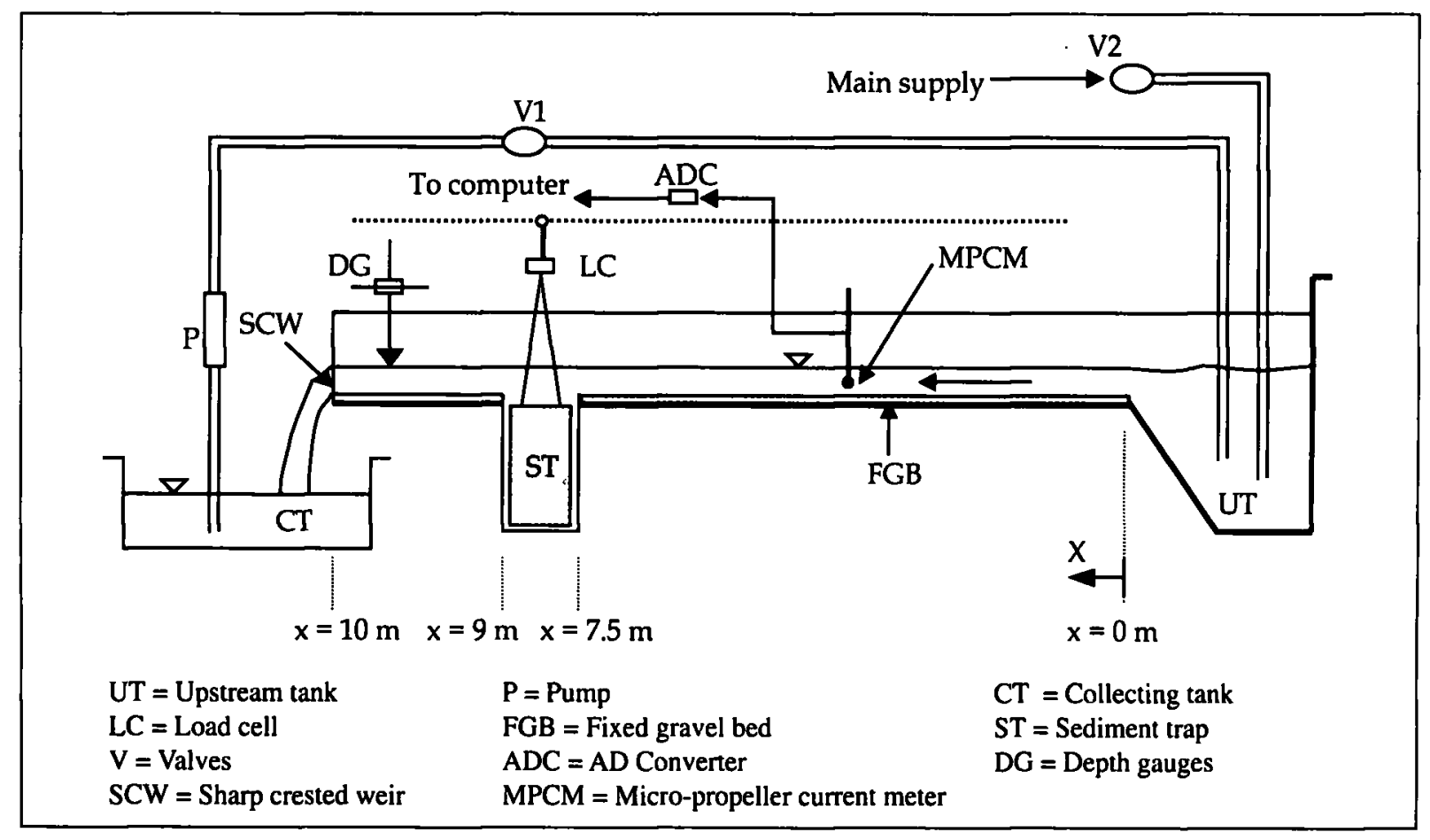

Figure 2 - Experimental set-up 
runs were conducted on a fixed rough bed for studying the bed shear stress due to steady, uniform flows.

\section{Experimental Procedure}

Natural river sand was sieved and the grains that passed through an $8 \mathrm{~mm}$ sieve and retained on a $2.3 \mathrm{~mm}$ sieve were used for the experiments. The mean diameter of sediment is $4 \mathrm{~mm}$ and the grain size distribution of sediments is shown in Figure 3 . The fixed bed was prepared by gluing a single layer of sediments on top of a plywood sheet with cement slurry and this sheet was fixed on to the channel bed.

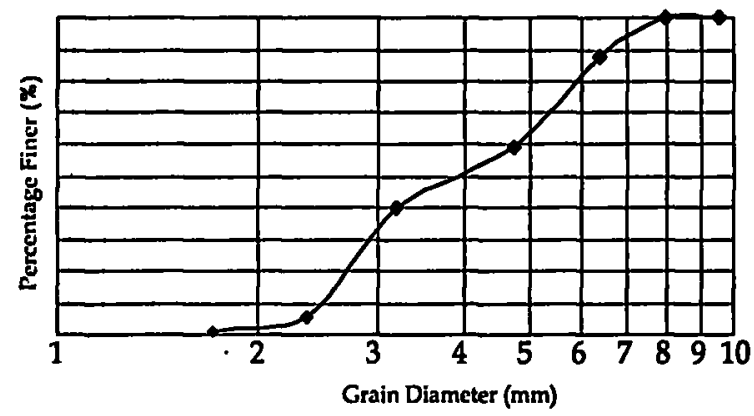

Figure 3 - Grain size distribution of sediments

The experiments were conducted over the fixed bed by generating four different discharges, namely, 3.5, 8.2, 12.0 and $24.0 \mathrm{l} / \mathrm{s}$ and each discharge was passed over four different channel slopes, $0.0055,0.0080,0.0110$ and 0.0135 . For each case, the velocity profiles were measured at every $0.5 \mathrm{~m}$ interval from $1 \mathrm{~m}$ to $7 \mathrm{~m}$ stretch from the channel entrance. Flow velocities were measured at mid-vertical of the flow cross section in order to minimize side-wall effects. The velocities were measured by a $5 \mathrm{~mm}$ diameter micro-propeller current meter coupled with an $A D$ converter and the velocity data were stored in the computer with the help of a computer programme. The readings were recorded at a $50 \mathrm{~Hz}$ frequency for 30 seconds per point. Based on these observations, it was found that the fully developed flow was established by the section at $5 \mathrm{~m}$ from the channel entrance. Accordingly, the velocity profiles measured at $5.0 \mathrm{~m}, 5.5 \mathrm{~m}$ and $6.0 \mathrm{~m}$ were taken for the present analysis. Water depths were also recorded at a section $5.5 \mathrm{~m}$ downstriam from the channel flow entrance. The summary of hydraulic parameters of the test cases is given in Table 1.
Table 1 - Summary of hydraulic parameters of test runs

\begin{tabular}{|l|c|l|l|l|l|}
\hline $\begin{array}{l}\text { Run } \\
\text { no. }\end{array}$ & S & $\begin{array}{c}\mathbf{Q} \\
(\mathbf{l} / \mathrm{s})\end{array}$ & $\begin{array}{c}\mathbf{y} \\
(\mathrm{cm})\end{array}$ & $\begin{array}{c}\mathbf{R}_{\mathrm{h}} \\
(\mathrm{cm})\end{array}$ & $\mathbf{F r}$ \\
\hline S1Q1 & 0.0135 & 24.0 & 8.1 & 7.59 & 0.90 \\
S1Q2 & 0.0135 & 12.0 & 5.3 & 5.10 & 0.80 \\
S1Q3 & 0.0135 & 8.2 & 4.0 & 3.90 & 0.71 \\
S1Q4 & 0.0135 & 3.5 & 2.4 & 2.37 & 0.56 \\
\hline S2Q1 & 0.0110 & 24.0 & 8.7 & 8.14 & 0.79 \\
S2Q2 & 0.0110 & 12.0 & 5.7 & 5.47 & 0.71 \\
S2Q3 & 0.0110 & 8.2 & 4.1 & 3.98 & 0.68 \\
S2Q4 & 0.0110 & 3.5 & 2.5 & 2.47 & 0.53 \\
\hline S3Q1 & 0.0080 & 24.0 & 9.6 & 8.86 & 0.70 \\
S3Q2 & 0.0080 & 12.0 & 6.1 & 5.83 & 0.62 \\
S3Q3 & 0.0080 & 8.2 & 4.6 & 4.46 & 0.55 \\
S3Q4 & 0.0080 & 3.5 & 2.6 & 2.56 & 0.46 \\
\hline S4Q1 & 0.0055 & 4.0 & 10.3 & 9.34 & 0.62 \\
S4Q2 & 0.0055 & 12.0 & 7.1 & 6.70 & 0.54 \\
S4Q3 & 0.0055 & 8.2 & 5.1 & 4.91 & 0.48 \\
S4Q4 & 0.0055 & 3.5 & 3.1 & 3.04 & 0.40 \\
\hline
\end{tabular}

\section{Results and Discussion}

As a typical result, the velocity profiles measured at the $5.5 \mathrm{~m}$ section for four different slopes and discharges are illustrated in Figure 4.

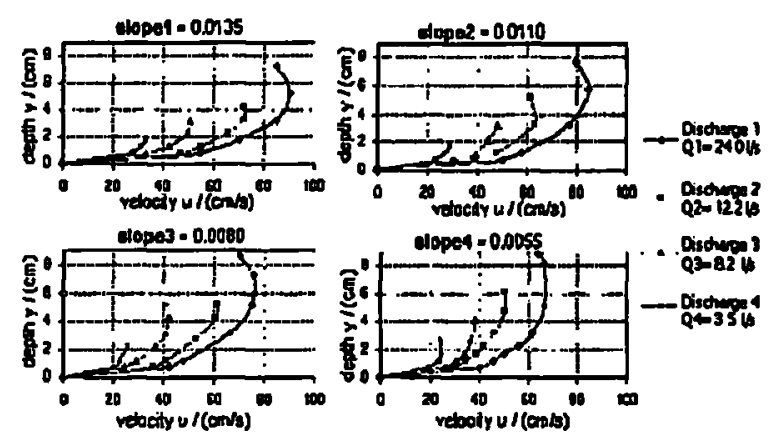

Figure 4 - Velocity profiles measured at $5.5 \mathrm{~m}$ section

Figure 5 illustrates the variation of $U / u^{*}$ with $R / V$ $k s$ for all test cases and the results are compared with the velocity profile given by log law using mean velocity of flow. The average values of $u^{*}$ and $\mathrm{k}_{\mathrm{s}}$ computed from methods 1 and 2 were used in this analysis. The experimental results are in a reasonable agreement with the theoretical velocity profile, suggesting that the experimental data are of good quality.

The location of reference bed level should be known for computing $u^{*}$ according to the 
method 1 described in Sec.(2). In this case, the definition of bed level is related to the velocity profile and it is located by extending the velocity profiles below the lowest velocity reading to reach zero velocity as indicated in Figure 1. According to the experimental results, it was found that the distance between the reference bed level and the top of the sand particles, $\Delta y$ vary between $4.5 \mathrm{~mm}$ and $5.8 \mathrm{~mm}$.

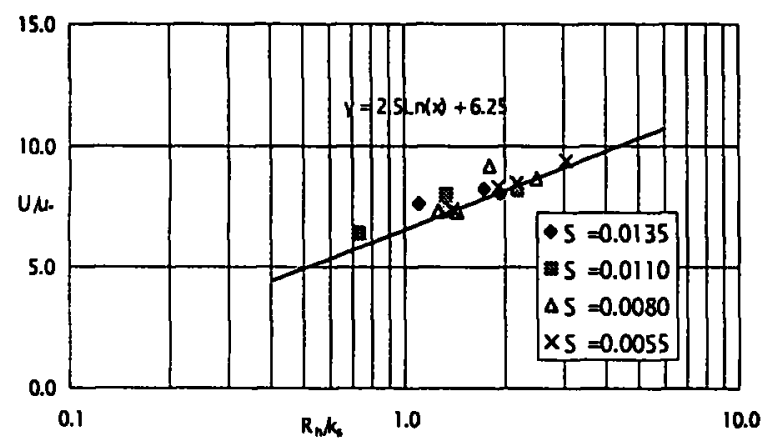

Figure 5 - Variation of $U / u$, with $R_{h} / k_{s}$

Figure 6 shows the variation of non-dimensional parameters $\Delta u / u^{*}$ with (uk/v). This graph shows a good correlation between $\Delta \mathrm{u} / \mathrm{u}^{*}$ and $(u k / v)$ irrespective of channel slopes. It can be seen that $\Delta \mathrm{u} / \mathrm{u}^{*}$ decreases with increasing $(u k / v)$. However, $\Delta \mathrm{u} / \mathrm{u}^{*}$ will not reach zero even with very high values of $(u k / v)$, suggesting that the reference bed level for the velocity profiles should lie below the top of granular material. This highlights the importance of considering the reference bed level for computation of shear velocity, as the actual bed level does not coincide with the top of the sand grains.

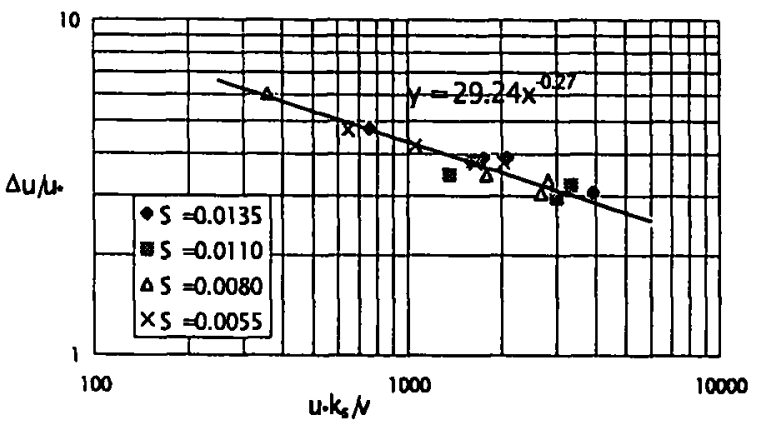

Figure 6 - Variation of $\Delta u u^{*}$ with $(u, k / v)$

Kabir [2] also presented a similar relationship between $\Delta \mathrm{u} / \mathrm{u}^{\star}$ and $(u k / \nu)$, however, his results appeared to vary with the channel slope. This discrepancy in results could have been due to the use of different ks values in his analysis however, this information was not explicitly stated in his description. In addition, most of his experimental runs were in the range of supercritical flow whereas, all test cases in the present study are with sub-critical flow.

The variation of $\Delta y / k s$ with $(u k / \nu)$ for different channel slopes is shown in Figure 7 . It is interesting to note that all the experimental data points fall on to a single curve irrespective of the channel slope, resulting in a functional relationship between $\Delta y / k s$ with $(u k / v)$. The non-dimensional parameter, $\Delta y / k s$ appeared to decrease sharply with low values of shear Reynolds numbers and reaching nearly 0.10 asymptotically for high shear Reynolds numbers. In general, $\Delta y / k s$ varies between 0.40 and 0.10 within the hydraulic parameters considered during this study. In comparison to this, Song et al. [8] suggested 0.25 for $\Delta y / k s$ when analysing velocity profiles in open channel flows on movable gravel beds. Although this value seems to be reasonable for $\Delta y / k s$ as an average value, it can also be as low as 0.10 if shear Reynolds numbers are very high. Therefore, it is recommended that Eq.(4) be used for computing $\Delta y$ in order to establish the reference bed level. However, the extrapolation of this equation for shear Reynolds numbers larger than 4000 is not recommended as the range beyond the shear Reynolds number of 4000 is not validated during this study.

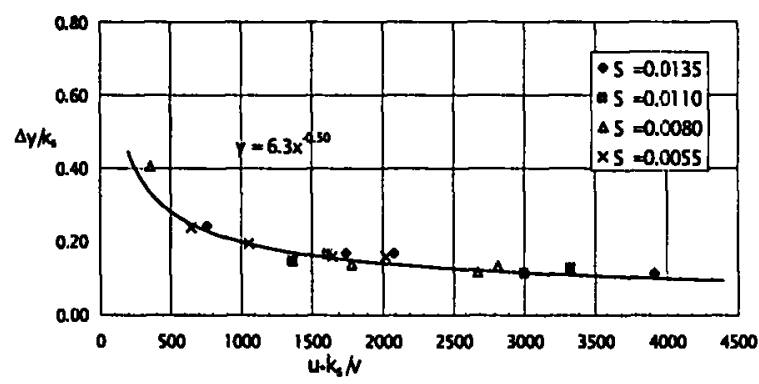

Figure 7 - Variation of $\Delta y / k s$ with $(n, k / v)$

$$
\frac{\Delta y}{k_{s}}=6.3\left(\frac{u_{*} k_{s}}{v}\right)^{-0.5} ; \frac{u_{*} k_{s}}{v} \leq 4000
$$

For each test case, the shear velocity was computed using four different methods presented in Sec.(2).

Figure 8 shows the variation of percentage differences in shear velocities given by different methods with $(u k / v)$ for all test runs. The shear velocities computed by methods (1) and (3) appear to be very close to each other and the maximum difference in shear velocities is about 
$5 \%$. The maximum percentage differences in shear velocities computed by method (2) with methods (1) and (3) are nearly $10 \%$ and $13 \%$, respectively. In contrast, the shear velocities given by method 4 are higher than those given by the other three methods indicating that the maximum percentage difference is as high as $45 \%$. However, it is interesting to note that the differences in shear velocities derived from all four methods seem to decrease with shear Reynolds number as shown in Figure 8.

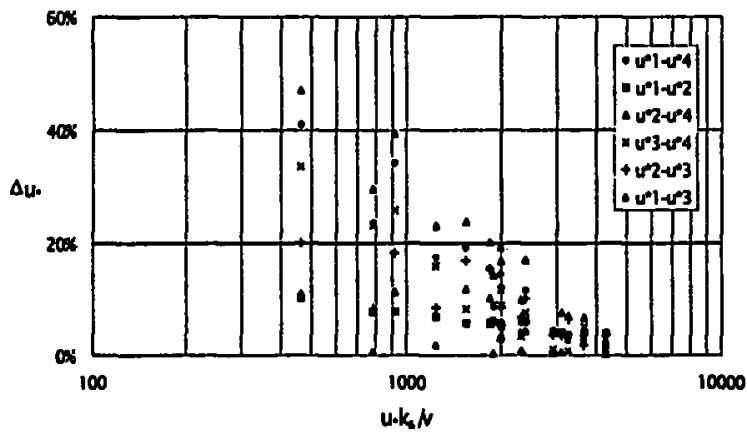

Figure 8 - Variation of change in shear velocities by different methods with $(u, k / v)$

The above analysis reveals that the choice of bed level, whether it is passing through sand grains at a height of average particle size or taking the hypothetical bed level where the velocity is absolutely zero, would make about $10 \%$ difference in shear velocities at low values of Reynolds numbers within the hydraulic parameters considered during this study. However, this difference could be much more pronounced if Reynolds number is further low. However, it is difficult to state which method is more accurate as the actual values of shear velocities to estimate bed shear stresses are not available. Although various methods are available for measuring bed shear stress in-situ, the application of such methods might not be that reliable on rough beds.

\section{Summary and Conclusions}

The effect of reference bed level for measurement of water depth on bed shear stress was analysed in detail using a series of laboratory experiments in steady, uniform open channel flows over a rough bed. Two metheds of treating the channel bed, one with the datum passing through sand grains at a height of average grain size and the other, taking the hypothetical bed level as the datum where the velocity becomes zero, were evaluated. Four different methods of estimating bed shear stress were compared. The following conclusions can be made:

(i) According to method 1, the difference between the hypothetical bed level and the top of the sand grains $(\Delta y)$ decreases sharply with low Reynolds numbers eventually reaching a constant value for high Reynolds numbers. A functional relationship was developed between $\Delta y / k s$ with $(u k / 0)$ which can be applicable for the shear Reynolds numbers less than about 4000 .

(ii) Shear velocity varies according to the choice of bed level and the difference in shear velocities computed using methods 1 and 2 varies nearly $10 \%$. This difference increases with low values of shear Reynolds numbers and vice versa.

(iii) Shear velocities predicted by method 4 are always greater than those predicted by the other three methods. However, the difference in shear velocities derived from all four methods seems to decrease with the shear Reynolds number. These differences are quantified for a range of Reynolds numbers.

\section{Acknowledgements}

The authors wish to acknowledge the financial support given by the National Science Foundation, Sri Lanka, Grant Number CRG/ $98 / \mathrm{P} / 02$ for conducting the experimental runs. The second author wishes to gratefully acknowledge the scholarship received from the Science \& Technology Personnel Development Project of the Ministry of Science \& Technology, Sri Lanka for his postgraduate studies.

\section{References}

1. Chang, H.H.: Fluvial Processes in River Engineering. John Wiley and Sons, Inc, 1988.

2. Chien N, Z. Wan: "Mechanics of Sediment Transport". The American Society of Civil Engineers, ASCE Press, 1998.

3. Graf W.H., Song T.: Bed-shear stress in nonuniform and unsteady open channel flows, J. Hydr. Res., 33(5), pp. 699 - 704, 1995. 
4. Henderson F.M.: Flood waves in prismatic channels, J. Hydr. Div., ASCE, 89(4), pp.39 67, 1996.

5. Kabir M.R.: Bed load transport due to unsteady flow, PhD Dissertation, Katholieke Universiteit Leuven, Belgium, 1993.

6. Kabir M.R., Trofs H.: Comparison of different methods to calculate bed shear stress, Wat. Sci. Tech., 25(8), pp.131-140, 1992.

7. Keulegan G.H.: Laws of turbulent flows in open channels, J. Res., Nat. Bureau of Standards, 21(6), pp.707-741, 1938.

8. Song T., Graf W.H., Lemmin U.: Uniform flow in open channels with movable gravel bed, J. Hydr. Res., 32(6), pp.861 - 876, 1995.

9. Tu H. and Graf W.H.: Friction in unsteady open-channel flow over gravel beds, J. Hydr. Res., 31(1), pp.99 - 110, 1993. 